\title{
ERZÄHLTE KULTURGESCHICHTEN ZU SKIDOO VON ALEX CAPUS
}

Nach dem großen Erfolg des Romans Léon und Louise (2011) veröffentlichte Alex Capus das nur 77 Seiten umfassende Buch mit dem Titel Skidoo und dem Untertitel „Meine Reise durch die Geisterstädte des Wilden Westens “. ${ }^{1}$ Das Buch umfasst eine Sammlung kleiner, skurriler Geschichten aus der Vergangenheit dieser Städte. Der ironische Ton, der zwar alle Texte Capus' prägt, in diesem aber noch besonders bedeutungstragend ist, wird schon auf dem Buchumschlag angekündigt, auf dem die Bemerkung des Autors zitiert wird: „Wenn ein Mann einen Liebesroman geschrieben hat, muss er hernach zum Ausgleich etwas Ordentliches tun. Einen Western schreiben zum Beispiel. Man ist sich das einfach schuldig, nicht wahr?" (Capus 2012) Die Aussage ironisiert in erster Linie die geschlechtliche Markierung von Genres - des Liebesromans als „weiblicher“ Gattung und des Westerns als „männlicher“ Gattung -, sie lenkt aber auch die Aufmerksamkeit auf die Frage der Gattungsbestimmung des Textes Skidoo selbst, in dem das für die meisten Texte von Capus typische erzählerische Spiel mit Fakten und Fiktion deutlich ersichtlich ist, da es durch Komprimierung und die Integrierung von Bildmaterial noch gesteigert wird. Eben dieses Spiel erschwert die Gattungszuordnung der meisten Werke von Capus, die deshalb oft als fiktionalisierte Biographien oder fiktionalisierte Reiseberichte bezeichnet werden (vgl. Herrmann 2017). Auch Skidoo weist Merkmale des Reiseberichts und der Biographie auf, worauf im Folgenden noch näher eingegangen wird, doch der Focus der vorliegenden Untersuchung soll sich auf eine Dimension des Textes richten, die bisher noch nicht untersucht worden ist und die von der These ausgeht, dass Skidoo als - schon seine Kürze und sein heiterer Ton verführen dazu - eine Art kulturgeschichtliches Scherzo bezeichnet werden kann. Zwar entspricht diese Bezeichnung keiner der anerkannten Literaturgattungen und dient somit nicht einer gattungstheoretischen Typisierung und literaturhistorischen Kategorisierung, sondern vor allem der Öffnung neuer

1 Der Beitrag ist im Rahmen des Forschungsprogramms Interkulturelle literaturwissenschaftliche Studien (Nr. P60265) entstanden, das von der Slowenischen Forschungsagentur aus öffentlichen Mitteln finanziert wird. 
Interpretationsmöglichkeiten. Sie ermöglicht es, den Text nicht nur im Kontext der zeitgenössischen fiktionalen Literatur zu lesen, sondern auch im Kontext kulturanalytischer und -geschichtlicher Werke, konkret in der Tradition jener Werke, die aus europäischer Perspektive einen kritischen Blick auf Amerika richten und gleichzeitig implizit oder explizit die Frage nach der Form solcher Reflexionen stellen. Gemeint sind hier vor allem Hector St. John de Crèvecœurs Briefe eines amerikanischen Farmers (1782), Alexis de Tocquevilles Über die Demokratie in Amerika (1835/1840), Jean Baudrillards Amerika (1984) und Tzvetan Todorovs Eroberung Amerikas (1982). Doch zunächst soll der Text im Hinblick auf seine unterschiedlichen Merkmale und Bestandteile - gemeint sind vor allem die Elemente der Biographie und des Reiseberichts sowie das Bildmaterial - näher dargestellt werden.

\section{BIOGRAPHIE}

Capus' Erzählverfahren, bei dem auf Grundlage genau recherchierter historischer Fakten fiktionale Geschichten konstruiert werden, ähnelt zwar den auf dem Buchmarkt stark vertretenen fiktionalisierten Biographien berühmter Persönlichkeiten, die in Romanform diese Persönlichkeiten vorstellbar und zugänglicher zu machen versuchen, wobei sie gleichzeitig entweder moralische, politische oder ideologische Lehren vermitteln oder schlicht den Voyeurismus und die Sensationslust des Lesepublikums bedienen. ${ }^{2}$ In drei Aspekten unterscheiden sich jedoch seine Texte wesentlich von diesem Genre, und zwar durch den ironischen Ton, die mehr oder weniger stark ausformulierte Metaebene und durch die Wahl historischer Persönlichkeiten, die zu literarischen Figuren gemacht werden. Durch den ironischen Ton, der durch die Selbstironie des Erzählers noch unterstrichen wird, werden die den Texten impliziten ethischen und politischen Standpunkte ständig in Frage gestellt, wodurch die belehrenden Tendenzen der fiktionalen Biographik unterlaufen werden. Die historischen Persönlichkeiten, die als Vorlage für seine literarischen Figuren dienen, sind keine Berühmtheiten, sondern bestenfalls bekannte Menschen, oft aber sogar solche, die in den Archiven kaum, im kulturellen Gedächtnis jedoch überhaupt keine Spuren hinterlassen haben. Diese Wahl unterläuft die Tendenz zu aufsehenerregenden Enthüllungen der Biographik und verhindert die Einordnung seiner Bücher in entsprechende Verkaufskategorien. Sie hat als solche auch politische, soziale und kulturelle Implikationen, die sich am Beispiel von Skidoo deutlich aufzeigen lassen. Schließlich integrieren seine Texte auch Hinweise auf die Fiktionalität, Überlegungen zur Schreibweise und Verweise auf die Unzuverlässigkeit und Lückenhaftigkeit des Erzählten.

2 Vgl. z. B. die exemplarische Studie von Marija Javor Briški zu den diversen aktuellen Literarisierungen von Martin Luther (Javor Briški 2018). 
Den Roman Reisen im Licht der Sterne. Eine Vermutung beendet Capus mit einem längeren Zitat aus einem Brief von Robert Louis Stevenson. Der Roman selbst begibt sich auf eine Reise auf den Spuren dieses Schriftstellers und seines Romans Die Schatzinsel, so dass das Zitat mit der erzählten Geschichte verknüpft ist, gleichzeitig aber ist es auch als autoreflexiver Kommentar auf Capus' im Roman angewandte Schreibstrategie zu lesen. Stevenson erklärt in dem zitierten Brief, warum er „Biografisches viel lieber als Fiktion“ (Capus, Reisen, 221) mag. Die Begründung, dass darin „der unlogische Widersinn des Lebens“ (ebd.) immer wieder auftauche, obwohl oder weil es nicht so ,abgeschlossen wie der mieseste aller Romane" sei, lässt sich auch auf Capus' Schriften übertragen. Die aus kausal zusammenhängendem Erzählen sich ergebende Abgeschlossenheit, die seit Aristoteles als konstitutives Merkmal eines sprachlichen Kunstwerks gilt, widerspricht in Stevensons Überlegungen der ebenfalls seit Aristoteles gültigen Forderung nach Glaubwürdigkeit, weil er sie im Bezug zum Leben sucht, während sie andererseits textintern begründet werden kann, d.h. in der Kohärenz der fiktiven Welt. Im explizit als Roman bezeichneten Text Der Fälscher, die Spionin und der Bombenbauer versucht Capus den „unlogische[n] Widersinn des Lebens“ in einer kohärenten fiktionalen Form auszudrücken, die gleichzeitig den Forderungen nach der Geschlossenheit und nach der Glaubwürdigkeit entgegenkommt. Dies gelingt ihm durch sparsame Anwendung bereits bekannter Erzählmittel und deren innovative Verquickung. Auf der thematischen Ebene wird durch die Geschichte Emile Gilliérons - er rekonstruiert aus Bruchstücken archäologischer Funde geschlossene Werke, wobei diese Rekonstruktionen zwangsläufig verfälschend sind - die Arbeit des Biographen reflektiert, der aus bruchstückhaften Daten Lebensgeschichten rekonstruiert, eben auch die Lebensgeschichte Gilliérons. Auf der Ebene der Motive erscheinen wiederholt Variationen der Motive von Gittern, Netzen und Netzwerken, die Querverbindungen zwischen den einzelnen Erzählsträngen schaffen und so den Zusammenhalt des Textes sichern. Gleichzeitig aber verweisen sie auf die Textur, also die netzartige Struktur von Texten im Allgemeinen und des erwähnten Romans im Besonderen, da in ihm die Verknüpfung dreier Lebensgeschichten die Vernetzung auch zum Gestaltungsprinzip dieses Textes macht.

Eine der Hauptfiguren im letztgenannten Roman ist Laura d'Oriano, die als eine im Spionagenetzwerk agierende Spionin auf die potentiell subversive Kraft derartig organisierter Handlungsträger verweist. Die literarisch umgestaltete Figur des Physikers Felix Bloch, die zweite Hauptfigur, bietet Capus jedoch die Möglichkeit, das Netz-Motiv auch auf anderen Ebenen zu entfalten. Bloch, der sich unter anderem mit Kristallgittern beschäftigte, inspiriert zu Phantasien, die bis zu einer gitterartigen Grundstruktur des Universums reichen. Gleichzeitig wird in der Erzählung die Bedeutung der Zusammenarbeit der jungen Physiker hervorgehoben, die sich ohne institutionellen Zwang vernetzen und dabei bahnbrechende Entdeckungen machen. Doch schon bald materialisieren sich die Netze und Gitter in dem Zaun, der sie in Los Alamos schützt und gefangen hält, um die Atombombe zu bauen (vgl. Capus 2013: 240). 
Capus verbindet in seinem Roman drei Geschichten literarisierter historischer Figuren zu einem lose verknüpften erzählerischen Netzwerk, in dem immer wieder explizit hervorgehoben wird, dass der Erzähler nicht alles weiß und vieles undeutlich und unbestimmt bleiben muss, wodurch auch darauf aufmerksam gemacht wird, dass der Großteil eines Netzes nicht aus Linien und Knoten besteht, sondern aus den Leerstellen zwischen ihnen. Für den Sinn des Textes sind die Leerstellen ebenso konstitutiv wie das Erzählte selbst, das sich vom Nicht-Erzählten (Ausgelassenen, Verschwiegenen, Unsagbaren) differenziert und es umschließt.

Durch solche Interventionen heben sich die Texte von Capus sowohl von Biographien wie auch von fiktionalisierten Biographien ab. In Skidoo ist es vor allem die Selbstironie des Ich-Erzählers, der über seine Recherchen zur Biographie des Bierbrauers Louis Munzinger aus Death Valley berichtet, die nicht nur das Schreiben von Biographien, sondern auch die Thematisierung und Selbstreflexion dieses Schreibens verfremdet. Der Ich-Erzähler versucht durch mühsame Recherchen zu beweisen, obwohl die vorgefundenen Dokumente die These nicht belegen können, dass Louis Munzinger ,wenn auch nicht direkt ein Sohn meiner Stadt, so doch ihr Ur-Ur-Ur-Ur-Großneffe sechsten Grades war. Gewesen sein könnte." (Capus 2012: 24) Der Prozess, in dem der Erzähler die Lücken in der Dokumentation verbissen aufzufüllen versucht, wird durch elliptische Sätze unterstrichen, die sein Scheitern eingestehen. Nachdem er seine Überzeugung äußert, „dass Louis eben doch ein Oltner gewesen sein muss“, folgt eine zögerliche Zurücknahme dieser Behauptung: „Ziemlich sicher. Sehr wahrscheinlich. Ausgeschlossen ist es jedenfalls nicht.“ (Capus 2012: 22) Das deklarierte Ziel dieser Bemühungen ist, die „Ahnengalerie“ seiner Freunde Pit und Katrin Munzinger, bei denen er sich ,alle paar Monate das Kopfhaar" schneiden lässt, zu erweitern (Capus 2012: 21). Damit wird nicht nur die einst dem Adel vorbehaltene, das Erbe von Macht und Reichtum bestimmende Ahnenforschung ironisiert, sondern auch das Leben der historisch weniger Bedeutenden aufgewertet. Dadurch wird die bereits erwähnte Konvention der (fiktionalen) Biographien unterlaufen und der politische Demokratisierungsprozess wörtlich genommen: Liegt in der Demokratie die Macht beim Volk, so verdienen auch die Bierbrauer und Frisöre die Aufmerksamkeit von Ahnenforschern und Biographen. Damit aber diese Aufmerksamkeit nicht zur Überheblichkeit und neuen Standesdünkeln führt, wird sie vom Erzähler bereits im Ansatz ironisch gebrochen.

\section{3}

\section{REISELITERATUR}

Der Untertitel von Skidoo - Meine Reise durch die Geisterstädte des Wilden Westens - wie auch die zahlreichen Abbildungen von Reisenden und von Verkehrsmitteln (von verschiedenen Kutschen bis zu Motorrädern) erlaubt es, das Buch zur Reiseliteratur zu zählen, doch der Begriff wird in der Literaturwissenschaft heute so weit gefasst, dass eine 
solche Zuordnung allein noch nicht viel zum Verständnis des Textes beiträgt. ${ }^{3}$ Schon Michail M. Bachtin unterschied in seinem Werk Chronotopos mehrere Typen literarischer Werke, die das Motiv des Weges variieren. Neben Abenteurerromanen und Werken, die einen Lebensweg nachzeichnen, also den Weg im übertragenen Sinne verstehen (vgl. Bachtin 2008, 21-57), hebt er vor allem den „geographischen Roman“, der über fremde Länder informiert (vgl. ebd.: 28), und den Schelmenroman (vgl. ebd.: 51, 180), der die verschiedenen sozialen Schichten eines Landes darstellt, hervor. Doch der Text Skidoo, wenn er auch Elemente all dieser Typen beinhaltet, lässt sich keinem von ihnen eindeutig zuordnen.

Eine aktuellere Typologie der zeitgenössischen Reiseromane des 21. Jahrhunderts wird von Leonhard Herrmann in seiner Studie Literarische Vernunftkritik im Roman der Gegenwart vorgeschlagen. Er unterscheidet Romane, in denen „die Reisen historischer Entdeckerfiguren fiktionalisiert" werden, zu denen er auch Romane von Alex Capus zählt (Herrmann 2017: 285), und Romane, in denen Reisen beschrieben werden, ,die der Autor oder die Autorin - so die paratextuelle Markierung - selbst erlebt haben will und nun in fiktionalisierter Form wiederzugeben in Anspruch nimmt“ (ebd.). „Das erstgenannte Verfahren verweist auf den (historischen) Gelehrtenroman, der seine Geschehensebene in enger Anlehnung an faktuale Erzählungen gestaltet, das letztgenannte auf das Genre des Reiseberichts, das ebenfalls durch eine große Nähe zwischen faktualen und fiktiven Gehalten markiert ist" (ebd.: 285).

Die von Herrmann vorgeschlagene Zuordnung trifft sicherlich für einige Romane und Erzählungen von Capus zu, z. B. den Roman Munzinger Pascha (1997) oder den Roman Eine Frage der Zeit (2007), Skidoo hingegen enthält Elemente beider Texttypen. So werden historische Entdeckerfiguren wie Francisco Vásquez de Coronado zwar erwähnt, doch werden sie nicht zu Helden der Erzählung gemacht. Coronado ist eine Figur in einem Abschnitt mit dem Titel Hawiku (Capus 2012: 57-62), doch die Helden dieser Geschichte sind vielmehr die Hopi, die ihn in die Irre führten und sich so ,weitere zweihundertfünfunddreißig Jahre Ruhe vor den spanischen Invasoren verschafft hatten“ (ebd.: 62). Die Geschichten verschiedener historischer Figuren, die nicht nur Entdecker sind, werden von der Geschichte des Ich-Erzählers eingerahmt, der die Reise durch die Geisterstädte gemacht haben will, was auch paratextuell markiert wird. Neben dem Untertitel, der darauf verweist, sind auch die Innenseiten des Umschlags mit einer Karte seiner Reise ausgestattet. Doch im Text wird nur an zwei Stellen kurz auf diese Reise eingegangen, ansonsten erfährt der Leser nichts über die Gegenwart des Reisenden oder die Gegenwart der bereisten Städte (es sind ja Geisterstädte), umso mehr aber über ihre Vergangenheit. In den Erzählungen über diese Vergangenheit zieht sich der Erzähler zurück und nimmt fast eine auktoriale Position ein, die durch den Bericht über den Fortlauf

3 Vgl. z. B. die sehr unterschiedlichen Texte, die im Sammelband Reiseliteratur der Moderne und Postmoderne (Holdenried et al. 2017) analysiert werden. 
seiner Recherchen jedoch gebrochen wird: Das Erzählte ist nicht das Wissen eines allwissenden Erzählers, sondern das Resultat eines recherchierenden Ich-Erzählers, der aus dem gefundenen Material auswählt, das Ausgewählte ordnet, artikuliert und durch Ironie implizit kommentiert. Die erste explizite Erwähnung seiner Reise macht der IchErzähler im ersten, einleitenden Absatz, in dem er seine Hingezogenheit zu Kleinstädten, die seiner Heimatstadt ähneln, erklärt. „Kürzlich geriet ich im Nordosten Kaliforniens hinter den verschneiten Bergspitzen der Sierra Nevada auf 2554 Metern über dem Meer in eine Goldgräber-Geisterstadt namens Bodie." (ebd.: 7) Schon in dieser Einleitung wird auf der Satzebene die Mischung von Fakten und Fiktion ersichtlich, da der Satz an den schematisierten Beginn von Märchen erinnert (zu einer unbestimmten Zeit, hinter den Bergen ...), gleichzeitig aber auch sehr genaue geographische Angaben beinhaltet. Die zweite konkrete Erwähnung der Reise ist im letzten Abschnitt zu finden, in dem er seinen Rückweg ,auf der vielbesungenen Route 66“ beschreibt: „Ach, es war ein niederschmetterndes Erlebnis“ (ebd.: 65). Die Strecke ist bereits in den 1960er Jahren durch die Fernsehserie Route 66 (1960-1964) und den Film Easy Rider (1969) berühmt geworden, mit zwei Werken, die das road genre begründeten, das nicht mit geographischen Romanen und Reiseberichten verwandt ist, sondern in der Tradition der Pikareske steht. Doch die Reisenden, die der Ich-Erzähler dort trifft - und hier geht er auf die Gegenwart und seine aktuellen Erfahrungen ein - sind keine modernen Easy Riders oder Picaros, sondern Touristen mit i-Phone und Bluthochdrucktabletten im Gepäck (vgl. ebd.: 66-67).

Skidoo ist mit zahlreichen Abbildungen ausgestattet (insgesamt 54 Bilder). Die größte Gruppe bilden dokumentarische Abbildungen historischer Gebäude und Fahrzeuge aus den USA, eine weitere große Gruppe bilden historische Portraits, dazu kommen aber auch andere Abbildungen, z. B. Faksimile alter Zeitungen. Die Funktion dieser Bilder ist, zumindest auf den ersten Blick, illustrativ, d. h. sie bilden das ab, worüber der Text erzählt, um ihn zu verdeutlichen, zu verifizieren und glaubwürdiger zu machen, aber ohne wesentliche zusätzliche Informationen beizusteuern. Die Illustration ist die gängigste Form der Kombination von Text und Bild in Werken, in denen der Text dominiert. Weniger verbreitet ist eine Integrierung von Bildern in den Text, bei der die Bilder den Text ergänzen oder konterkarieren. In diesen Fällen beziehen sich die Bilder nicht in erster Linie auf die Handlungsebene, wie im Fall der Illustration, sondern auf die thematische Ebene, die durch sie an Komplexität gewinnt (vgl. Virant 2013). Bei näherer Betrachtung erweist sich jedoch, dass die Bilder in Skidoo nicht nur die Funktion von Illustrationen erfüllen, sondern eben die thematische Komplexität des Werks erhöhen.

In dem Bericht über die extensiven Recherchen zum Stammbaum der Munzinger erwähnt der Erzähler auch Johann Adam, der „Kaiserlicher Reichposthalter in 
Bruchmühlbach war und im Herbst 1812 Kaiser Napoleon auf seinem Rückweg aus Moskau eine Nacht lang unter seinem Dach beherbergte" (Capus 2012: 22-23). Am Rand des Textes sind eine Vedute Bruchmühlbachs und ein Portrait Napoleons abgedruckt. Die Bilder steuern dem Text keine neuen Informationen bei, aber sie erhöhen auch nicht seine Glaubwürdigkeit. Sie mögen zwar die Faktizität der historischen Existenz des Ortes und des Kaisers unterstreichen, sie mögen auch die Vorstellungskraft des Lesers unterstützen, sie beweisen jedoch keineswegs, dass Napoleon wirklich unter dem Dach von Johann Adam Munzinger übernachtete. Das offensichtliche Scheitern der Bilder, das Erzählte zu verifizieren, wirkt ironisch nicht nur textintern in Bezug auf die erzählte Begebenheit, sondern ironisiert auch die gängigen Praktiken moderner Massenmedien, die z. B. neue Behauptungen durch Archivbilder aus völlig anderen Kontexten ausstatten, wodurch Visualität und Kontiguität genutzt werden um Faktizität und Kausalität zu suggerieren.

Ein Bild hebt sich von anderen verwendeten Bildern ab. Es zeigt einen kleinen Jungen in zu großen Strampelhosen, einem Munitionsgürtel über der Brust, skeptisch in die Kamera blickend (ebd.: 16). Wegen des zweifarbigen Drucks und der Heterogenität des Bildmaterials im Buch hebt es sich visuell nicht wesentlich von anderen Bildern ab, wenn davon abgesehen wird, dass er in der Halbtotale statt in der für den Western üblichen amerikanischen Einstellung gezeigt wird (wie z. B. das Bild von Hadji Ali, ebd.: 74). Was dieses Bild von anderen abhebt, ist die Betitelung: „Der Autor im Spätsommer 1964, irgendwo in der Normandie" (ebd.: 16). Der Abschnitt in der Erzählung, der durch dieses Bild offensichtlich illustriert wird, berichtet über die eigenen Erfahrungen des Erzählers mit dem Leben am Rande oder gar außerhalb der Gesellschaft:

Ich selbst habe in meinem Leben nur einmal der Gesellschaft von Frauen und Kindern entsagt - das war im Spätsommer 1964 -, um allein, frei und auf mich selbst zurückgeworfen ein Leben als gesetzloser Desperado hinter der Brombeerhecke zu führen. Schon nach einem halben Tag aber musste ich das Unternehmen abbrechen, weil ich erkannte, dass die Gesellschaft von Regenwürmern, Schnecken und Ameisen mich auf Dauer nicht ganz ausfüllen würde. (ebd.: 16)

Das Bild des Jungen, das angeblich den Autor zeigt, der im Spätsommer 1964 tatsächlich drei Jahre alt war, hat hier mindestens zwei Funktionen. Erstens verknüpft es nicht nur die zwei Ebenen von „faktualen und fiktiven Gehalten“ (Herrmann 2017: 285), sondern gleich drei Ebenen: die des Autors als historischer Persönlichkeit, des vermittelnden Erzählers und die der erzählten Figuren. Der so konstituierte autodiegetische Erzähler wird jedoch gleichzeitig ironisiert: Auch er ist fehlbar, obwohl der Fehler des erzählten Ich, den er hier eingesteht, zeitlich bereits entfernt ist. Die zweite Funktion dieses Bildes zeigt sich in der Verbindung mit dem letzten Abschnitt, in dem er über seine aktuelleren Erfahrungen auf der Route 66 berichtet, und bezieht sich auf die thematische 
Ebene des Textes. Die Desperados und Outlaws, die in dem letzten Abschnitt thematisiert werden und sowohl Helden des Genres Western wie auch des Road Movie sind, verkörpern den Wunsch nach Freiheit und Individualität, die durch diese Genres auch zum Kern des amerikanischen Traumes erhoben werden. Dieser Traum wird jedoch bereits im ersten Abschnitt von dem Erzähler als infantile Wunschvorstellung entlarvt und abgetan, während die heutigen wohlsituierten Touristen auf der Route 66 noch immer in ihm verfangen sind. In den dazwischenliegenden Abschnitten werden skurrile Geschichten erzählt: von dem verirrten Entdecker Coronado, von Räubern und Mördern, von dem Bierbrauer Munzinger, der eine „Elf- oder Zwölfjährige“ (ebd.: 20) heiratete, oder vom Erfinder Jonathan Newhouse, der in seiner Rüstung, die er als Schutz vor der Hitze in der Wüste erfunden hatte, schließlich erfror (vgl. ebd.: 47-53). Es sind keine Geschichten von glorreichen Entdeckern und Eroberern. Sie erzählen nicht über Erfolg und verwirklichte Träume, sondern über Gier, Verbrechen und Dummheit, schließlich aber auch über das Scheitern, denn die Städte, die einst von den Protagonisten gegründet worden sind, sind bereits untergegangen.

Der Erzähler moralisiert nicht und verurteilt die Protagonisten dieser Geschichten nicht, er entzieht ihnen aber eine privilegierte Position, und zwar durch die Aneinanderreihung vieler Geschichten und durch die durchgängige Ironisierung. Zudem setzt er ihnen positive Gegenbilder entgegen. Munzinger wird z. B. nicht als pädophil bezeichnet, obwohl seine Beziehung zu der Elf- oder Zwölfjährigen heute diese Bezeichnung verdienen würde. Statt einer solch expliziten Verurteilung fokussiert er sich auf das Mädchen und fragt sich nach ihren Lebensumständen, die er jedoch nicht ergründen kann, „weil über Frauen zu jener Zeit selten etwas in der Zeitung stand“" (ebd.: 20). Als positive Gegenbilder erscheinen auch die amerikanischen Ureinwohner, die weder verallgemeinert noch individualisiert, sondern nach Stammeszugehörigkeit differenziert werden. Sie werden als klug, erfinderisch und gelassen dargestellt, wie in der bereits erwähnten Episode mit Coronado. Während Naturkatastrophen wie Erdbeben und Hochwasser die Städte der Siedler zerstörten, wurden die Shoshone in ihren Tipis „durch das Beben nur kurz aufgeweckt und schliefen dann weiter" (25). Nachdem die zerstörte Stadt endgültig verlassen wird, kehren sie, „die seit Jahrtausenden im Surprise Canyon gelebt hatten“ wieder zurück. Zwar werden in der Darstellung der Ureinwohner der Genozid und ihre heutigen sozialen Probleme ausgeblendet, doch es wird auch kein klischeehaftes Bild der verschwindenden edlen Wilden vermittelt, sondern ein positives Bild des Überlebens in Zeiten des Postkolonialismus. Hermes zählt Capus zu den deutschsprachigen Autoren, die beginnen, den wilhelminischen Kolonialismus literarisch aufzuarbeiten (vgl. Hermes 2017: 260). Skidoo kann ebenfalls als ein Beitrag zur Aufarbeitung des Kolonialismus, der Kolonisierung Amerikas, gelesen werden, in der die Deutschen zwar nicht die Hauptrolle spielten, aber doch beteiligt waren. So hebt der Erzähler bereits am Anfang hervor: „Ich stelle mir den Schrecken der ersten Goldgräber von 1849 vor, die noch nie im Leben einen leibhaftigen Bären gesehen hatten, weil sie wenige Monate zuvor noch 
Straßenbahnschaffner in Nürnberg oder Polizeiwachtmeister in Paderborn oder Bauernknecht in Niederbipp gewesen waren" (Capus 2012: 14).

Die Portraits Napoleons und Hitlers, die am Rande der Geschichte über den Bierbrauer Munzinger, der den Wilden Westen erobert, abgedruckt sind, erfüllen nicht nur eine illustrative Funktion, indem sie sich konkret auf das Erzählte beziehen, sondern tragen auch zur thematischen Komplexität bei, da sie den kleinen Mann mit seinem kleinen Eroberungszug in den Kontext der großen historisch-politischen Bewegungen des Expansionismus, Imperialismus und Kolonialismus stellen. Dieser thematische Bogen, also die kritische Auseinandersetzung mit der Rolle der Deutschen bei der Kolonisierung Amerikas, wird im letzten Abschnitt bis in die Gegenwart gespannt, denn die Touristen, die heute die Route 66 in Besitz nehmen, sind „Fliesenleger, Architekten und Wirtschaftsprüfer aus Brunsbüttel, Friedrichshafen oder Herzogenbuchsee“ (ebd.: 65-66).

\section{5}

\section{KULTURGESCHICHTEN}

Die Anlehnung an „faktuale Erzählungen“, die Herrmann bei dem Typus der Reiseliteratur beobachtet, zu dem er auch Werke von Capus zählt (285), lädt ein, Skidoo auch im Kontext solcher „faktualen Erzählungen“ zu lesen, und zwar in diesem Fall konkret im Kontext von Texten, die sich aus europäischer Perspektive kritisch mit Amerika auseinandersetzen und bereits eine lange Tradition haben. Schon St. John de Crèvecœur wählte in seinen Briefen eines amerikanischen Farmers (Letters from an American Farmer) einen fiktiven Erzähler, um seine dokumentarischen, stellenweise kritischen Beobachtungen der amerikanischen Lebensweise zu vermitteln. Neben der Kritik an der im Süden praktizierten Sklaverei, richtet sich seine Kritik auch gegen das Verhalten der Siedler im Hinterland:

But to return to our back settlers. [...] The chase renders them ferocious, gloomy, and unsociable $[\ldots]$ That new mode of life brings along with it a new set of manners, which I cannot easily describe. These new manners being grafted on the old stock, produce a strange sort of lawless profligacy, the impressions of which are indelible. (St. John de Crèvecœur 1951: 51-52)

Die Kinder dieser Siedler bekämen keine Bildung und folgten nur den Vorbildern der Eltern, heißt es (vgl. ebd.). Im letzten Kapitel wird die Kritik verallgemeinernd auf die europäische bzw. die euro-amerikanische Gesellschaft - ,the fictitious society in which we live" (ebd.: 215) - gerichtet. Als Beweis dafür, dass seine Kritik berechtigt sei, erzählt er über die vielen Europäer, die freiwillig mit den Indianern lebten und ihre Lebensweise angenommen hätten, während umgekehrt kein Fall bekannt sei: ,we have no examples of even one of those Aborigines having from choice become Europeans!“ (ebd.). Das 
Leben in der Wildnis der Neuen Welt befördert den Verfall der Sitten der Siedler, sofern sie überhaupt welche aus Europa mitbrachten, denn, so Capus in Skidoo, über neunzig Prozent waren männlich und zwischen 18 und 25 Jahre alt (vgl. Capus 2012: 18).

Neben der Kritik am Kolonialismus und dem konkreten Verhalten der großen und kleinen Eroberer der Neuen Welt, die noch expliziter in Tzvetan Todorovs Buch Die Eroberung Amerikas. Das Problem des Anderen (La Conquête de l'Amérique: La Question de l'autre) formuliert wird, werden diese kulturgeschichtlichen Werke über Amerika von der indirekten, kritischen Auseinandersetzung mit Europa geprägt. Jean Baudrillard eröffnet sein Buch Amerika (Amérique) mit dem englischen Satz: „Caution: objects in this mirror may be closer than they appear!“ (Baudrillard 2004: 9). Amerika dient dem Europäer als Spiegel, in dem er seine eigene Kultur betrachten kann. Die Kritik, die Baudrillard dabei an Amerika übt, ist in diesem Fall auch eine Kritik an Europa. An späterer Stelle verneint Baudrillard ausdrücklich die Möglichkeit dieser Art von Reflexion:

In Wirklichkeit gewinnt man hier nicht, wie ich gehofft hatte, Distanz zu Europa, man bekommt keinen neuen Blickwinkel. [...] Es geht nicht darum, einen kritischen Standpunkt zu Europa einzunehmen. Den kann man sich in Europa selbst aneignen. Und was bliebe im übrigen zu kritisieren, was nicht schon tausendfach kritisiert wurde? (Baudrillard 2004: 45)

Trotz dieser Behauptung sind Europa und die Europäer in diesem Buch ständig explizit (vgl. ebd.: 31, 38, 45) oder implizit anwesend. Implizit unter anderem durch seinen Bezug auf Tocqueville (vgl. ebd.: 126). Tocqueville behält in seinem Buch Über die Demokratie in Amerika (De la démocratie en Amérique), in dem er die Vor- und Nachteile sowie die Gefahren der Demokratie erörtert, ständig Europa, vor allem Frankreich, im Blick, und prüft die Übertragbarkeit von demokratischen Strukturen nach Europa. In Skidoo gibt es keine derartigen direkten Vergleiche zwischen Europa und Amerika, wie auch verallgemeinernde Feststellungen über Europa und Amerika vermieden werden. Bereits im ersten Satz wird der Focus auf das Kleine und Konkrete gerichtet, gesehen von einem im Kleinen und Konkreten verankerten Blickpunkt: Der Erzähler blickt auf Kleinstädte, die ihn ,an meine Heimatstadt Olten erinnern“ (Capus 2012: 7), was durch eine Fotografie von Olten unterstrichen wird. So wird ein Vergleich auch auf diese Ebene projiziert: „Bodie ist umgeben von Kleinstädten, die von Kleinstädten umgeben sind, die einander zum Verwechseln ähnlich sehen, das war im Wilden Westen nicht anders als im Schwarzwald, in der Toskana oder im Alpenbogen“ (ebd.: 13). Dass diese Ähnlichkeit nicht zufällig ist, wird durch die wiederholten Verweise auf die Herkunft der Siedler deutlich, die mit den städtebaulichen Lösungen auch die Kleinstadtmentalität in die Neue Welt mitbrachten. Die implizite Kritik richtet sich nicht in erster Linie gegen diese Mentalität selbst, auch wenn sie ironisiert wird, sondern gegen die kolonialistische Ideologie, die ihre Übertragung erst ermöglichte. 
Interessanter als der Vergleich auf inhaltlicher Ebene ist jedoch die Frage nach der Schreibweise und den Methoden. Wie oben erwähnt, vermeidet Capus verallgemeinernde Aussagen über ganze Staaten, Staatengemeinschaften oder Kontinente, wenn jedoch Vergleiche in kleineren Dimensionen angestellt werden, werden sie ironisch gebrochen. Schon Tocqueville äußert im zweiten Teil seiner Überlegungen zu Amerika seine Vorbehalte gegenüber Generalisierungen:

Les idées générales n'attestent point la force de l'intelligence humaine, mais plutôt son insuffisance, car il n'y a point d'êtres exactement semblables dans la nature: point de faits identiques; point de règles applicables indistinctement et de la même manière à plusieurs objets à la fois. (Tocqueville 2012: 399)

Gleichzeitig aber stellt er generalisierend die unterschiedliche Affinität zu solchen Generalisierungen bei Franzosen, Engländern und Amerikanern fest und räumt ein, dass Generalisierungen schnelles Beurteilen komplexer Zusammenhänge ermöglichen, obwohl auf Kosten der Genauigkeit (vgl. ebd.). In diesem Bewusstsein liefert er immer wieder Beispiele, mit denen er seine Behauptungen verdeutlicht, obwohl er bei diesen Beispielen nur selten auf das Erzählen konkreter Geschichten zurückgreift, die er als eigene Erinnerungen oder als ihm erzählte Erinnerungen in der Form eines Ich-Erzählers vermittelt. ${ }^{4}$ Todorov hingegen findet eine Form, bei der er sowohl auf Verallgemeinerungen als auch auf die subjektive Sicht eines Ich-Erzählers verzichtet, bis auf den Epilog, in dem er seine Vorgehensweise reflektiert:

La forme de discours qui s'est imposée à moi pour ce livre, l'histoire exemplaire, résulte encore du désir de dépasser les limites de l'écriture systématique, sans pour autant « revenir » au mythe pur. A comparer Colon et Cortés, Cortés et Moctezuma, je prends conscience de ce que les formes de la communication, production comme interprétation, même si elles sont universelles et éternelles, ne sont pas offertes au libre choix de l'écrivain, mais qu'elles sont corrélées aux idéologies en vigueur, et peuvent par là même en devenir le signe. Mais quel est le discours approprié à notre mentalité hétérologique? Dans la civilisation européenne, le logos a vaincu le mythos; ou plutôt: à la place du discours polymorphe, deux genres homogènes se sont imposés: la science et tout ce qui lui est apparenté relève du discours systématique; la littérature et ses avatars pratiquent le discours narratif. [...] Je ne pouvais me séparer de la vision

4 So z. B. in zwei kurzen Geschichten, mit denen er die Unterschiede in der Mentalität der drei Rassen in Amerika verdeutlichen will. Eigene Erfahrung: „Je me souviens que, parcourant les forêts qui couvrent encore l'État d'Alabama, je parvins un jour auprès de la cabane d'un pionnier.“ (Toqueville 2012: 310). Vermittelte Erfahrung: ,je fis connaissance avec un officier américain, le major H., qui, un jour, [...] me raconta le fait suivant: J'ai connu autrefois $[\ldots]$ ] (ebd.). 
des « vainqueurs » sans renoncer en même temps à la forme discursive qu'ils s'étaient appropriée. J'éprouve le besoin [...] d'adhérer au récit qui propose plutôt qu'il n'impose; de retrouver, à l'intérieur d'un seul texte, la complémentarité du discours narratif et du discours systématique [...]. Certains faits que je rapporte conduisent à des affirmations générales; d'autres (ou d'autres aspects des mêmes faits), non. A côté des récits que je soumets à l'analyse, restent d'autres, insoumis. Et si, en ce moment même, je « tire la morale » de mon histoire, ce n'est nullement en pensant livrer et figer son sens ; un récit n'est pas réductible à une maxime; mais parce que je trouve plus franc de formuler quelques-unes des impressions qu'elle laisse sur moi, puisque je suis aussi l'un de ses lecteurs. (Todorov 1982: 159-160)

Todorov verwendet nicht das Begriffspaar faktual/fiktiv, das von Herrmann und anderen zeitgenössischen Literaturwissenschaftlern verwendet wird, um die Verfahren moderner Reiseliteratur zu beschreiben, sondern greift zunächst zurück auf das altgriechische Begriffspaar logos/mythos und unterscheidet dann den systematischen und den narrativen Diskurs, wobei er die ideologische Markierung dieser Diskurse hervorhebt. Obwohl die drei Begriffspaare eng verwandt sind, gibt es zwischen ihnen doch Bedeutungsunterschiede, die selbst ideologisch sein können. Das Begriffspaar Fakt/Fiktion suggeriert zwar vor allem die Unterscheidung von wirklich/unwirklich, doch wird es oft auch mit der Unterscheidung von wahr/unwahr verbunden. Fakten entsprechen dabei der Wirklichkeit und der Wahrheit, während der Fiktion als Erfindung der Zugang zu einer höheren Ebene der Wahrheit, die dem mythos noch zugesprochen wurde, verweigert wird. Im Begriffspaar systematischer/narrativer Diskurs sind die Verhältnisse zur Wirklichkeit und zur Wahrheit nicht im Begriff selbst etymologisch angelegt. Vor allem aber schließt der narrative Diskurs Fakten nicht a priori aus. So wird die Bevorzugung des systematischen Diskurses als relevanter, wenn nicht gar einziger Form, in der die Wahrheit ausgesprochen wird, als ideologisches Konstrukt deutlicher. Diese Bevorzugung schreibt Todorov den „Siegern“ zu, also den Eroberern und Kolonisatoren. Der systematische Diskurs ist, so die Konsequenz seiner Ausführungen, der Ort, an dem die Wahrheit der Sieger ausgesprochen wird. Seine Weigerung, sich vollkommen dem systematischen Diskurs zu unterwerfen, hat also ideologische Gründe. Er weigert sich jedoch auch eine Polarisierung zu akzeptieren, in der das Narrative als einzige Möglichkeit bleibt, die Wahrheit der Besiegten auszusprechen. Sein Versuch, einen Diskurs zu entwickeln, in dem sich das Systematische und das Narrative ergänzen, ist mehr als nur eine Vermischung zweier Diskurse. Es ist der Versuch einer Aufhebung der Polarisierung in ihrer Synthese.

Wird Capus' Text Skidoo nicht durch die Optik der Opposition von Fakt und Fiktion betrachtet, sondern durch Todorovs Differenzierung zwischen systematischem und narrativem Diskurs sowie deren ideologischen Implikationen, so wird das in ihm verwendete 
Verfahren auch deutlicher in seiner antikolonialen Haltung lesbar. Schon bei dem Verhältnis zwischen Autor und Ich-Erzähler können so unnötige Verstrickungen vermieden werden, die durch das Verhältnis zwischen dem faktualen Autor und dem fiktionalen Ich-Erzähler entstehen, da der letztere zwar erfunden ist, aber doch Ähnlichkeiten mit dem Autor aufweist und doch so Anteil am Faktualen zu haben scheint. Im Rahmen der Todorov'schen Differenzierung ist der Ich-Erzähler eine narrative Kategorie, die nicht dem Autor gegenüber steht, sondern einem systematischen Diskurs, der objektiv sein will, also bevorzugt ohne einen Erzähler, vor allem aber ohne ein „Ich“, formuliert wird. Durch die Synthese der beiden Diskurse wird jedoch auch die Erzähltheit des systematischen Diskurses sichtbar und sein Erzähler, der sich im reinen systematischen Diskurs verleugnet, erweist sich als ein auktorialer Erzähler, der auf der Selbstverleugnung seine Autorität aufbaut. Eben dieser Prozess wird in Skidoo durch die Thematisierung der Recherche und die Selbstironie des Erzählers unterlaufen.

Todorov bezeichnet sein Verfahren als exemplarische Geschichte (,l'histoire exemplaire"; Todorov 1982: 159), wobei das Exemplarische in beiden Bedeutungen des Wortes „Beispiel“ zu verstehen ist: als vorbildhaftes und als illustratives Beispiel. Er analysiert historische Quellen und arbeitet daraus Beispiele heraus, die über die Namen-, Gesichts- und Geschichtslosen der Kolonialgeschichte erzählen. Dadurch werden sie zwar nicht zu Subjekten der Weltgeschichte, aber doch zu Protagonisten eigener Lebensgeschichten. Die Vorbildfunktion der Geschichte betrachtet Todorov jedoch mit Skepsis: „A ignorer l'histoire, dit l'adage, on risque de la répéter ; mais ce n'est pas parce qu'on la connaît qu'on sait ce qu'il faut faire.“ (ebd.: 160). Wenn die Kenntnis der Geschichte zwar nicht unbedingt hilft, in der Zukunft das Richtige zu tun, so hilft sie mit ihren konkreten Beispielen doch zur Selbsterkenntnis:

Mais leur histoire peut être exemplaire pour nous parce qu'elle nous permet de réfléchir sur nous-mêmes, de découvrir les ressemblances comme les différences : une fois de plus la connaissance de soi passe par celle de l'autre. (ebd.)

Das Schreiben über Amerika als exemplarische Geschichte liefert keine Spiegelung des ganzen Kontinents Europa im Kontinent Amerika, sondern ermöglicht individuelle Selbsterkenntnis über die Geschichten der anderen, eine Selbsterkenntnis, die Voraussetzung für das Abwägen des zukünftigen Handelns ist.

Abschließend lässt sich zusammenfassen, dass die Rekontextualisierung von Capus' Werk und sein Vergleich mit kulturgeschichtlichen bzw. kulturanalytischen Werken über Amerika es möglich macht, den literaturwissenschaftlichen Fokus von dem Verhältnis zwischen Fakten und Fiktion auf die diversen möglichen Formen der Diskursivierung zu verschieben, wodurch sich andere Bedeutungsebenen erschließen lassen. Alex Capus' Text Skidoo kann so als eine exemplarische Geschichte des Wilden Westens gelesen werden, die in narrativer Form mit Ironie die Lebensgeschichten kleiner Eroberer auffächert, 
die von Gier, Verbrechen und Dummheit geprägt sind und die letztendlich nicht von Erfolg, sondern von ihrem Scheitern als Gründerväter der heutigen Geisterstädte erzählen. Die Geschichten entwerfen weder eine Kulturgeschichte Amerikas noch ein Spiegelbild des Lesers oder der europäischen Kultur, auch lassen sie sich nicht auf eine Maxime reduzieren, aber sie bieten die Möglichkeit, die Art und Weise zu hinterfragen, wie Kultur- und Lebensgeschichten erzählt, von welchen Ideologien sie getragen werden und wie viel Illusion und Selbstbetrug sie instituieren. Ein kulturgeschichtliches Scherzo ist es also in doppelter Hinsicht: es vermittelt auf eine leichte, humorvolle Weise Geschichten über die Kulturgeschichte, wie peinlich sie auch sein mag, und stellt sie als Instrument nationaler Identitätsstiftung durch Ironie gleichzeitig in Frage. Wie Todorov es für seinen polymorphen Diskurs wünscht, zwingt die Erzählweise von Capus keine Erkenntnisse auf, sondern bietet die Möglichkeit von Selbstreflexion an.

\section{BIBLIOGRAPHIE}

BACHTIN, Michail M. (2008) Chronotopos. Frankfurt a. M.: Suhrkamp.

BAUDRILLARD, Jean (2004) Amerika. Berlin: Matthes \& Seitz.

CAPUS, Alex (2012) Skidoo. Meine Reise durch die Geisterstädte des Wilden Westens. München: Hanser.

CAPUS, Alex (2007) Reisen im Licht der Sterne. Eine Vermutung. München: BTB.

CAPUS, Alex (2013) Der Fälscher, die Spionin und der Bombenbauer. München: Hanser.

HERMES, Stefan (2017) Kolonialliteratur. D. Göttsche, A. Dunker, G. Dürbeck (Hg.), Handbuch Postkolonialismus und Literatur. Stuttgart: Metzler.

HERRMANN, Leonhard (2017) Literarische Vernunftkritik im Roman der Gegenwart. Stuttgart: Metzler.

HOLDENRIED, Michaela/Alexander HONOLD/Stefan HERMES (Hg.) (2017) Reiseliteratur der Moderne und Postmoderne. Berlin: Erich Schmidt.

JAVOR BRIŠKI, Marija (2018) Das Bild Luthers in der Literatur des 21. Jahrhunderts. Vestnik za tuje jezike X/1, 9-24.

ST. JOHN DE CRĖVECEUR, Hector (1951) Letters from an American Farmer. London: J. M. Dent/New York: E. P. Dutton.

TOCQUEVILLE, Alexis de (2012) De la démocratie en Amérique. Paris: Institut Coppet. TODOROV, Tzvetan (1982) La Conquête de l'Amérique : La Question de l'autre. Paris: Seuil.

VIRANT, Špela (2013) Bilder als integraler Bestandteil des literarischen Textes: zu Nikotin von Gregor Hens. B. Kadzadej (Hg.), Die Sprache im Bild - das Bild in der Sprache. Oberhausen: Athena, 80-84. 


\section{POVZETEK}

\section{Upovedana kulturna zgodovina: Skidoo Alexa Capusa}

Besedilo Skidoo švicarskega pisatelja Alexa Capusa je genološko težko umestiti, prav zato pa je primerno za eksemplarično analizo in ponazoritev specifičnih značilnosti njegovega literarnega ustvarjanja. Prispevek najprej poudari tiste značilnosti tega kratkega besedila, ki pripoveduje o popotovanju prvoosebnega pripovedovalca po ameriškem zahodu, ki se skladajo z ustaljenimi vzorci literariziranih biografij in potopisov, nato pa izpostavi tiste značilnosti, ki odstopajo. Nato obravnava razmerje besedila in slikovnega gradiva ter v sklepnem delu Skidoo umesti v kontekst izbranih kulturnozgodovinskih del, ki, podobno kot Capus, iz evropske perspektive opisujejo Ameriko: to so dela Hectorja St. John de Crèvecœura, Alexisa de Tocquevilla, Jeana Baudrillarda in Tzvetana Todorova. Primerjava teh del pokaže različne možne oblike diskurziviranja kulturne zgodovine. Upoštevanje tega vidika pa omogoča vpogled v doslej prezrte pomenske plasti Capusevega dela. Pri tem se izkaže, da je koncept »eksemplarične zgodovine« zelo primeren tudi za analizo dela Skidoo. Ta koncept mogoča, da Caupuseve zgodbice beremo kot upovedano kulturno zgodovino, ki ne slavi osvajalcev novega kontinenta, temveč osvetli zgodovino posameznikov, ki so to zgodovino ustvarjali, pri tem pa ne zamolči njihovih osebnih napak in porazov.

Ključne besede: sodobna nemška književnost, Alex Capus, biografija, potopis, kulturna zgodovina

\section{ABSTRACT}

\section{Narrated cultural history: Skidoo By Alex Capus}

The article focusses on the text Skidoo: A Journey Through the Ghost Towns of the American West, by the Swiss author Alex Capus, in order to point out some specific features of his literary work. The first part of the article shows those features of this short literary text that are congruent with the set schemes of the literary biography and travelogue, and underlines those that differ from them. It continues with an analysis of the relation between the text and the pictures integrated in the book. In the final part, Skidoo is compared to selected works that speak about the cultural history of America from a European point of view, starting with Hector St. John de Crèvecœur's Letters from an American Farmer, continuing with texts by Alexis de Tocqueville, Jean Baudrillard and Tzvetan Todorov. This comparison shows the different forms of discourse used in these works, and makes it possible to consider aspects of Skidoo that are easily overlooked, if only traditional genre categories are applied. In particular, Todorov's concept of l'histoire exemplaire, applied to Skidoo, makes it possible to read Capus' stories as part of a narrative cultural history that does not celebrate the conquest of a continent, but elevates the individual with all his faults and failures.

Keywords: modern German literature, Alex Capus, biography, travelogue, cultural history 